\title{
Identification and characterization of large DNA deletions affecting oil quality traits in soybean seeds through transcriptome sequencing analysis
}

\author{
Wolfgang Goettel ${ }^{1} \cdot$ Martha Ramirez $^{2} \cdot$ Robert G. Upchurch $^{2}$. \\ Yong-qiang Charles An ${ }^{1}$
}

Received: 2 November 2015 / Accepted: 28 April 2016/ Published online: 14 May 2016

(C) The Author(s) 2016. This article is published with open access at Springerlink.com

\begin{abstract}
Key message Identification and characterization of a 254-kb genomic deletion on a duplicated chromosome segment that resulted in a low level of palmitic acid in soybean seeds using transcriptome sequencing.

Abstract A large number of soybean genotypes varying in seed oil composition and content have been identified. Understanding the molecular mechanisms underlying these variations is important for breeders to effectively utilize them as a genetic resource. Through design and application of a bioinformatics approach, we identified nine coregulated gene clusters by comparing seed transcriptomes of nine soybean genotypes varying in oil composition and content. We demonstrated that four gene clusters in the genotypes M23, Jack and N0304-303-3 coincided with large-scale genome rearrangements. The co-regulated gene clusters in M23 and Jack mapped to a previously described 164-kb deletion and a copy number amplification of the Rhgl locus, respectively. The coordinately down-regulated gene clusters in N0304-303-3 were caused by a $254-\mathrm{kb}$
\end{abstract}

Communicated by H. T. Nguyen.

Electronic supplementary material The online version of this article (doi:10.1007/s00122-016-2725-z) contains supplementary material, which is available to authorized users.

Yong-qiang Charles An

yong-qiang.an@ars.usda.gov

1 US Department of Agriculture, Agricultural Research Service, Midwest Area, Plant Genetics Research Unit, Donald Danforth Plant Science Center, 975 N. Warson Rd, St. Louis, MO 63132, USA

2 US Department of Agriculture, Agricultural Research Service, Soybean and Nitrogen Fixation Research, 2417 Gardner Hall, Raleigh NC, 27695, USA deletion containing 19 genes including a fatty acyl-ACP thioesterase B gene (FATBla). This deletion was associated with reduced palmitic acid content in seeds and was the molecular cause of a previously reported nonfunctional FATB $1 a$ allele, $f a p_{n c}$. The M23 and N0304-304-3 deletions were located in duplicated genome segments retained from the Glycine-specific whole genome duplication that occurred 13 million years ago. The homoeologous genes in these duplicated regions shared a strong similarity in both their encoded protein sequences and transcript accumulation levels, suggesting that they may have conserved and important functions in seeds. The functional conservation of homoeologous genes may result in genetic redundancy and gene dosage effects for their associated seed traits, explaining why the large deletion did not cause lethal effects or completely eliminate palmitic acid in N0304-303-3.

\section{Introduction}

Soybean (Glycine max) is a dual purpose crop. The demand for soybeans is mainly driven by the high value of oil in dietary and industrial use and the protein that makes soybean meal a valuable commodity (Clemente and Cahoon 2009). With the increasing demand for soybean oil as an industrial resource for uses such as bio-fuel and greater consumer awareness of health issues related to dietary fats (Durrett et al. 2008), development of new soybean varieties with desirable fatty acid composition becomes a critical goal for genetic improvement of soybean. Commodity soybeans accumulate about $20 \%$ oil in their seeds, which contains on average $13 \%$ palmitic acid (16:0), $4 \%$ stearic acid (18:0), $20 \%$ oleic acid (18:1), $55 \%$ linoleic acid (18:2), and $8 \%$ linolenic acid (18:3) (Goettel et al. 2014; 
Pham et al. 2010). The main enzymes and metabolic pathways responsible for producing fatty acids in oilseed species are largely described (Voelker and Kinney 2001). In soybean, great efforts have been made to identify and characterize mutant alleles and to genetically engineer genes encoding enzymes involved in fatty acid biosynthesis for the improvement of oil composition (Clemente and Cahoon 2009; Wilson 2012).

Soybean is an allotetraploid species with a complex genome. The soybean genome has undergone two wholegenome duplication events that occurred 13 and 59 million years ago (Schmutz et al. 2010). It is estimated that about $75 \%$ of genes are retained in multiple copies. Many of the duplicated genes have conserved protein functions (Lin et al. 2010; Roulin et al. 2012). The two whole genome duplications created genetic redundancy and dosage effects for many phenotypic traits in soybean, and make genetic engineering for fatty acid composition more complicated and less predictable. Enzymatic reactions in the fatty acid metabolic pathway are often determined by a combination of more than two homoeologous genes (Pham et al. 2010). For example, the delta-twelve fatty acid desaturase 2 enzymes (FAD2) are encoded by a multi-gene family. The FAD2-2 sub-family, which consists of three genes, is mainly expressed in soybean vegetative tissues while the two microsomal FAD2-1 genes [FAD2-1A (Glyma10g42470) and FAD2-1B (Glyma20g24530)] are mainly expressed in developing seeds. It has been shown that soybean genotypes carrying both mutated alleles of $F A D 2-1 A$ and $F A D 2-1 B$ have an average of $82-86 \%$ oleic acid content, which is significantly higher than that of soybean genotypes containing each individual mutated allele (Pham et al. 2011). Understanding the contribution of each homoeologous gene to each enzymatic activity in soybean seeds facilitates the design of effective breeding strategies towards improvement of the soybean seed fatty acid profile.

Soybean oil with lower palmitic acid content offers substantial health benefits, as consumption of palmitic acid has been shown to increase the risk of developing cardiovascular diseases. A number of soybean genotypes containing low palmitic acid levels have been identified. They provide a rich genetic resource to breed new cultivars with low palmitic acid content. Some of their underlying loci (mostly referred to as "fap") have been identified. Fap alleles associated with reduced palmitic acid levels are $\mathrm{fap}_{1}$ in genotype C1726 (Cardinal et al. 2014; Erickson et al. 1988), $\mathrm{fap}_{3}$ in A22 (De Vries et al. 2011; Fehr et al. 1991; Schnebly et al. 1994), fap* in ELLP2 (Stijšin et al. 1998), sopl in genotype J3 (Rahman et al. 1996; Takagi et al. 1995) and fap $_{n c}$ in genotype N792077-12 (Burton et al. 1994; Cardinal et al. 2007; Wilson et al. $2001 \mathrm{~b}, \mathrm{c})$. With the exception of $f_{a p}$, a naturally occurring mutation, all other fap alleles were developed by chemical mutagenesis or by X-ray irradiation. While $f a p_{n c}$ is allelic with $f a p_{3}, f a p 1, f a p_{3}$, and $f a p^{*}$ represent independent genetic loci. sopl is not allelic to $f_{a p}$, and its allelism with other fap alleles has not been reported. The genes associated with $\mathrm{fap}_{1}$ and $\mathrm{fap}_{3}$ have been identified. $f_{l}$ is an allele of KASIIIA (Glyma09g41380) that encodes a 3-ketoacyl-ACP synthase enzyme III (Cardinal et al. 2014). A single nucleotide mutation that disrupts the exon1-intron1 splice junction of KASIIIA results in a truncated KASIIIA enzyme. $f_{a} p_{3}$ is an allele of the FATB 1a gene, which codes for a 16:0-acyl carrier protein (ACP) thioesterase enzyme (De Vries et al. 2011). fap $_{3}$ has a non-synonymous substitution, which produces a detrimental effect on the FATB1a function. $f_{a p}$ is a second mutant allele of FATB Ia. Southern, northern and cDNA analysis failed to detect FATBIa in genotypes containing the fap $_{n c}$ allele, suggesting that FATBla is deleted in $f a p_{n c}$ (Cardinal et al. 2007; Wilson et al. 2001a, c). However, the genome structural change underlying $\mathrm{fap}_{n c}$ has not been illustrated.

The ultimate goal of soybean seed fatty acid composition improvement is to develop cultivars containing a desirable fatty acid profile without negative impact on other soybean traits. For the design of an effective crossing and selection strategy, which fully utilizes the genetic resources, it is important to (1) identify and precisely define the genome structural changes underlying each mutant allele, (2) understand the functional redundancy of homoeologous genes and their contribution to fatty acid profiles, and (3) illustrate the impact of mutant alleles on other traits at molecular and systems levels. Availability of next-generation sequencing technologies enables us to sequence transcriptomes of soybean seeds, which simultaneously reveals two functional attributes of expressed genes, transcript sequence and accumulation levels (Goettel et al. 2014; Ozsolak and Milos 2011). Furthermore, comparative transcriptome analysis can effectively identify transcript sequence and expression variation of mutated genes among different germplasm. Their impact on expression of all other genes can be assessed at a systems level, which provides an insight into their potential interactions with other agronomic traits. This method is especially beneficial for the analysis of polyploid genomes since transcript sequences and accumulation levels of all homoeologous genes can be evaluated simultaneously to predict the contribution of each individual gene to their combinatorial protein activity (Goettel et al. 2014).

Recently, we applied next-generation sequencing technology to sequence seed transcriptomes from nine soybean genotypes varying in oil content and composition, and showed that genetic variation results in the expression change of thousands of genes (Goettel et al. 2014). To identify and characterize large structural genome rearrangements that affect the fatty acid composition of soybean seeds we 
further explored our transcriptome sequencing data. We developed a bioinformatics approach to identify several co-regulated gene clusters, and demonstrated that some of them are caused by large-scale genome segment deletions and amplifications. We identified a novel 254-kb deletion in genotype N0304-303-3 that includes the FATB1a gene encoding a fatty acyl-ACP thioesterase B. N0304-303-3 contains a reduced level of palmitic acid and also has altered levels of other major fatty acids (Goettel et al. 2014) (Suppl. Figure 1). We showed that the low palmitic acid content in N0304-303-3 was highly associated with the large deletion, suggesting that loss of FATB1a activity likely reduces palmitic acid in N0304-303-3. The deletion is located in a duplicated genome segment retained from the Glycine-specific whole genome duplication. Most genes in the deleted region shared a high similarity with their homoeologous genes in their expression patterns and protein sequences, which may have led to genetic redundancy and dosage effects of those homoeologs. We propose that loss of those genes due to the deletion event does not cause lethal and null phenotypes in N0304-303-3 because their homoeologous genes completely or partially complement their function.

\section{Materials and methods}

\section{Plant genotypes and PCR analysis}

Soybean [Glycine $\max$ (L.) Merrill] seeds for PI 904061, PI 92567, N69-2774, and N79-2077-12 were received from the USDA Soybean Germplasm Collection (http:// www.ars-grin.gov/npgs/). Seeds for the remaining genotypes were provided by Dr. Joe Burton (USDA-ARS in Raleigh, NC). Genomic DNA was isolated from $100 \mathrm{mg}$ of mature seed tissue using the DNeasy Plant Mini kit (Qiagen, Valencia, CA). PCR amplification reactions were conducted with the Expand High Fidelity Plus system from Roche (Mannheim, Germany) in a $25 \mu$ volume containing $50 \mathrm{ng}$ genomic DNA, $200 \mathrm{nM}$ each primer, $2.5 \mathrm{mM} \mathrm{MgCl}$, and $200 \mu \mathrm{M}$ deoxyribonucleotide phosphates (dNTPs). Amplification condition was 1 cycle at $94{ }^{\circ} \mathrm{C}$ for $4 \mathrm{~min}$, followed by 30 cycles at $94{ }^{\circ} \mathrm{C}$ for $30 \mathrm{~s}$, primer annealing temperature for $30 \mathrm{~s}$, and $72{ }^{\circ} \mathrm{C}$ for $30 \mathrm{~s}$. A final extension was conducted at $72{ }^{\circ} \mathrm{C}$ for $15 \mathrm{~min}$. For sequencing, amplicons were cloned into vector pCR2.1 with the TOPO TA cloning kit supplied by Invitrogen (Carlsbad, CA). Cloned fragments were sequenced at the Iowa State University Biotechnology Center, Ames, IA. A primer pair was designed to amplify an 805-bp product from $G$. $\max$ N0304-303-3 in the region of chromosome 5 flanking the $254-\mathrm{kb}$ deletion (forward primer GCCAGCTTAATGATCAGTTT, reverse primer TAGAACCACTCAGTGTTCTTTG, annealing temperature $57{ }^{\circ} \mathrm{C}$ ). Another primer pair that amplifies a 530-bp region of FATB1a (Glyma05g08060) was designed to be used in conjunction with the amplification reactions above (forward primer CAAGTGGACACTTGGGTTTC, reverse primer GCCTCCGTGTTAGCTTATTC, annealing temperature $61^{\circ} \mathrm{C}$ ).

\section{Seed fatty acid composition analysis}

Samples consisting of ten seeds each were ground to a powder using a small mortar and pestle under liquid nitrogen. Fatty acid methyl esters (FAMEs) of a subsample from each seed pool were prepared by acid methanolysis (Burkey et al. 2007). Frozen and ground seed tissue was heated to and held at $85{ }^{\circ} \mathrm{C}$ for $90 \mathrm{~min}$ in a $5 \% \mathrm{HCl}-95 \%$ methanol solution. FAMEs were partitioned two times into hexane and transferred to $2-\mathrm{ml}$ vials for analysis. The FAMEs were separated by gas chromatography using an HP 6890 GC (Agilent Technologies, Inc., Wilmington, DE) equipped with a DB-23 $30 \times 0.53 \mathrm{~mm}$ column (Agilent Technologies, Inc.). Operating conditions were $1-\mu \mathrm{l}$ injection volume, a 20:1 split ratio, and He carrier gas flow of $6 \mathrm{ml} \mathrm{min}{ }^{-1}$. Temperatures were 250,200 , and $275^{\circ} \mathrm{C}$ for the injector, oven, and flame ionization detector, respectively. Peak areas of the chromatograms were analyzed using HP ChemStation software. Fatty acid contents, as percentages, were calculated as $\mathrm{mg}$ fatty acid $\mathrm{g}^{-1}$ oil.

\section{Bioinformatic analyses}

Seed transcriptome sequencing data from nine genotypes previously generated in our laboratory were used in this study (Goettel et al. 2014). RNA-seq data for the nine soybean genotypes are available under NCBI-GEO series accession no. GSE56297. The transcript accumulation for each gene was normalized and indicated as Fragments Per Kilobase of transcript per Million mapped reads (FPKM) as previously described (Goettel et al. 2014). A gene with a mean FPKM value of all examined genotypes higher than 0.5 or with FPKM values higher than 0 in all examined genotypes was identified as transcribed. The normalized accumulation values of each gene were used to calculate their $Z$ scores as following:

$Z$ score $=(\mathrm{x}-\mu) / \sigma$

where $x=\log 2\left(\right.$ Sample $\left._{(\text {FPKM }+1)}\right)$

$\mu=\left(\sum_{\text {sample } 1 \ldots \text { sample } n} \log 2\left(\right.\right.$ Sample $\left.\left._{(\mathrm{FPKM}+1)}\right)\right) / n$

where $n$ is the total number of samples, $\sigma$ is the standard deviation of $\mu$.

A custom Perl script was developed to identify co-regulated genome regions within a genotype that contained four 
Table 1 Co-regulated gene clusters identified in nine genotypes

\begin{tabular}{lllllllll}
\hline No. of adjacent genes & Jack & FAM94-41 & M23 & N0304-303-3 & R02-6268F & R05-591 & R08-1450 & Total \\
\hline Down-regulated $^{\mathrm{a}}$ & & & & & & & & \\
4 & 0 & 0 & 0 & 0 & $\mathbf{1}$ & 0 & 0 & 1 \\
7 & 0 & 0 & 0 & $\mathbf{2}$ & 0 & 0 & 0 & 2 \\
18 & 0 & 0 & $\mathbf{1}$ & 0 & 0 & 0 & 0 & 1 \\
Total & 0 & 0 & 1 & 2 & 1 & 0 & 0 & 4 \\
Up-regulated $^{\mathrm{b}}$ & & & & & & & & \\
4 & $\mathbf{1}$ & 0 & 0 & 0 & 0 & $\mathbf{1}$ & 0 & 2 \\
5 & 0 & 0 & 0 & 0 & 0 & 0 & $\mathbf{1}$ & 1 \\
7 & 0 & $\mathbf{1}$ & 0 & 0 & 0 & 0 & 0 & 1 \\
8 & 0 & 0 & 0 & 0 & 0 & 0 & $\mathbf{1}$ & 1 \\
Total & 1 & 1 & 0 & 0 & 0 & 1 & 2 & 5 \\
\hline
\end{tabular}

a Down-regulated genes with $Z$ scores $\leq-2$

${ }^{\mathrm{b}}$ Up-regulated genes with $Z$ scores $\geq 2$ or more adjacent and transcribed genes each with a $Z$ score less than or equal to -2 or more than or equal to +2 . The regions identified were categorized as putative large deletions or amplifications for further validation. $Z$ scores of all differentially transcribed genes were displayed as a heat map in their chromosomal gene order.

The deleted genome sequences were used as queries in BLASTN searches to identify their homoeologous regions in the soybean genome. FASTA sequence files, GFF annotation files and comparison files generated by BLASTN were used as input files for the Artemis Comparison Tool (ACT) (Carver et al. 2005) to compare the duplicated soybean sequences and analyze their syntenic relationship.

MEGA5 (Tamura et al. 2011) was used for the evolutionary comparison of all homoeologous genes in the duplicated regions. Coding sequences of homoeologous genes were first aligned by ClustalW. Numbers of synonymous and non-synonymous substitutions per site were then calculated using the Nei-Gojobori model (1986). All positions containing gaps and missing data were eliminated.

\section{Results}

\section{Prediction of large segment deletions and amplifications based on transcriptome sequencing}

In a recent study, we sequenced the seed transcriptomes of nine soybean genotypes that vary in oil composition and content and identified a set of transcript SNPs and small indels (Goettel et al. 2014). Here, we developed a computational method to identify co-regulated gene clusters and predict large genome segment deletions and amplifications by comparing transcriptome sequencing data from nine genotypes. In our approach, we used the $Z$ score to measure expression differences among the genotypes for each gene. The genomic regions containing adjacent genes within a genotype with positive or negative $Z$ score values for each transcript above or below a threshold indicates that those genes are coordinately up-regulated or down-regulated. Genomic regions containing adjacent down-regulated genes with little or no transcript accumulation are indicative of putative deletions. We applied the algorithm in the analysis of the nine genotypes and identified four genomic regions in three genotypes (M23, N0304-303-3 and R02-6268F) that each contained more than three adjacent down-regulated genes with a $Z$ score value of $\leq-2$ (Table 1 ). The genomic region in M23 mapped to a previously identified 164-kb deletion on chromosome 10 (Bolon et al. 2011; Rahman et al. 1994). The two co-regulated regions in N0304-303-3 were caused by a 254-kb deletion (see below). Additionally, we identified five genomic regions in four genotypes (Jack, FAM94-41, R05591 and R08-1450) that each contained more than three adjacent up-regulated genes with $Z$ score values of more than or equal to 2 (Table 1 ). The coordinately up-regulated genome region in Jack coincided with the Rhgl locus (for resistance to $\underline{H}$ eterodera glycines), which is composed of four adjacent genes (Glyma18g02580, Glyma18g02590, Glyma18g02600 and Glyma18g02610). This finding is consistent with our previous report showing the expression of the four genes at the Rhgl locus was tenfold up-regulated due to a tenfold genome segment amplification in Jack (Goettel et al. 2014). Thus, this computational algorithm could be used effectively to both identify genome regions containing coordinately regulated genes and to predict large structural genome variation such as deletions and amplifications.

\section{Discovery and characterization of a novel 254-kb genomic deletion in N0304-303-3}

The two down-regulated genomic regions in N0304303-3 were separated by one down-regulated gene with a 


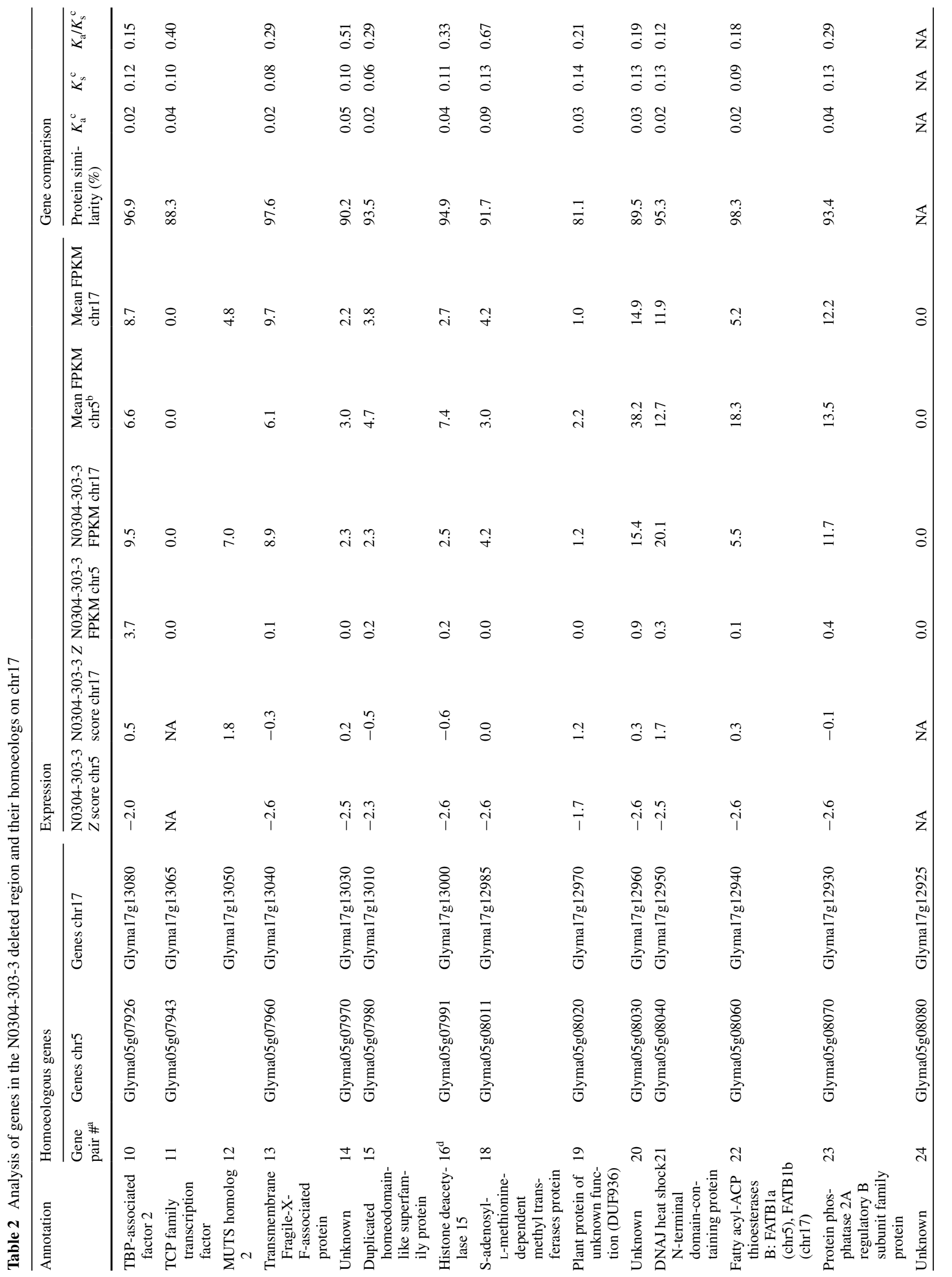







a

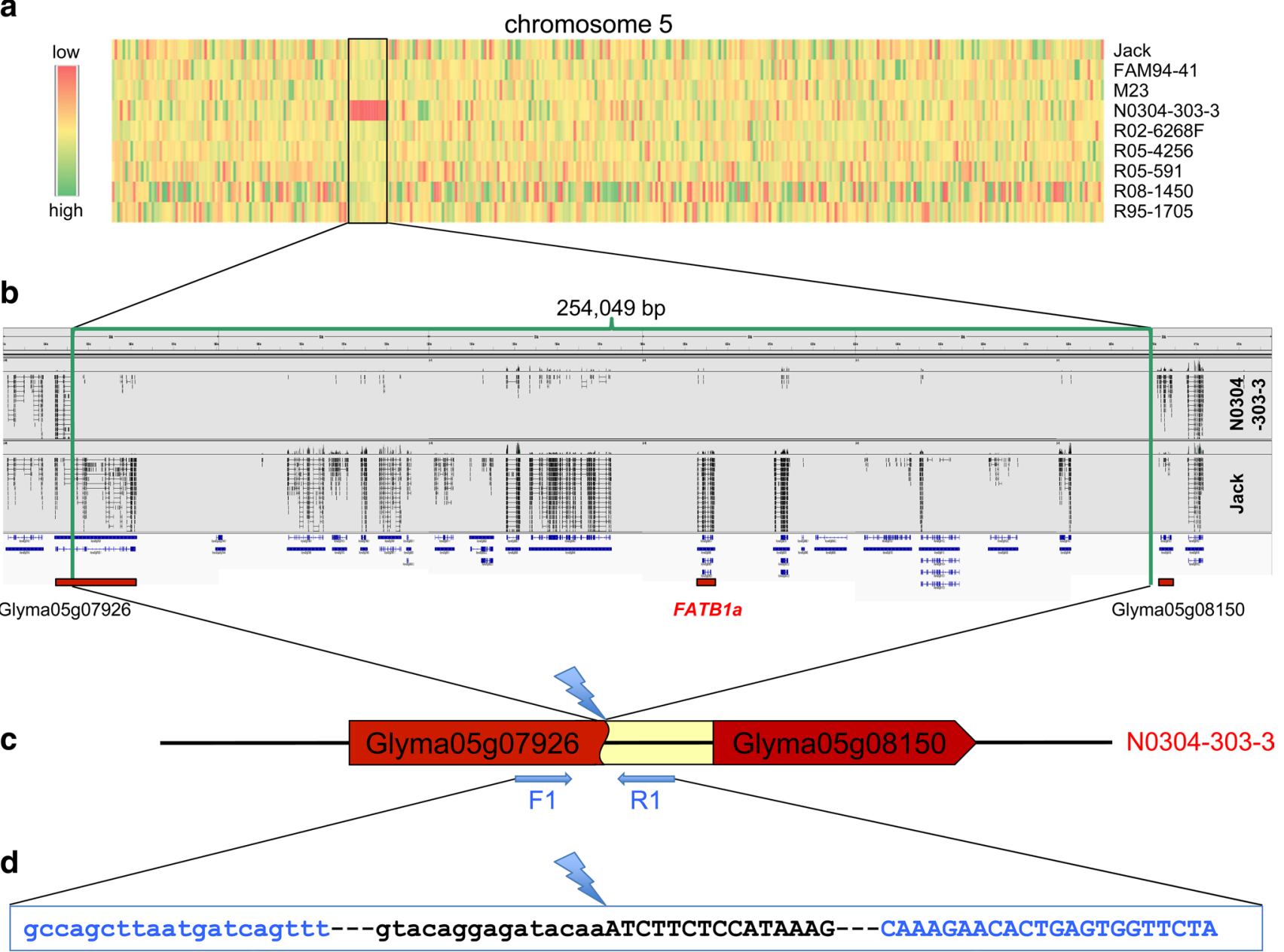

Fig. 1 Identification of a 254-kb deletion on chromosome 5 in N0304-303-3. a This heat map represents expression variation among nine soybean genotypes. The $Z$ score values of all genes arranged by chromosome location were displayed using a color gradient. Please note the red cluster of adjacent genes in N0304-303-3 that appear to be expressed at a significantly lower rate. These genes are listed in Table 2. b An IGV view of the region on chromosome 5, which contains the N0304-303-3 deletion, shows RNA sequence read alignments of soybean genotypes N0304-303-3 and Jack, and corresponding gene models. The deletion breakpoints, which are indicated by green bars, were determined by PCR analysis and sequencing of the amplification product (see below). The deletion in N0304-303-3 is

$Z$ score value of -1.7 . Therefore, the two down-regulated regions likely represent two sub-regions of a single large down-regulated genome segment in N0304-303-3. This region mapped to chromosome 5 and contained 18 genes (Table 2). Fourteen of the 18 genes were expressed in soybean seeds of the other analyzed genotypes, but none of them were transcribed at a significant level in N0304-3033, except for Glyma05g07926 located at one border of the genome region (Fig. 1a; Table 2). While a large number of RNA reads in N0304-303-3 aligned to the first eight exons of Glyma05g07926, only very few reads, which probably
254,049 bp in size and includes 19 gene models. The FATB1a gene, and the genes located in or next to the breakpoints are marked by red bars. c The deletion in N0304-303-3 generated a fusion gene that consists of the $5^{\prime}$ end of Glyma05g07926 (in red), a small intergenic sequence (in yellow) and Glyma05g08150 (in red). The blue arrows represent both PCR primers used for the amplification across the deletion site (not drawn to scale). d The partial sequence of the PCR product spanning the deletion junction is presented. The primer binding sites are depicted in blue. The $5^{\prime}$ region flanking the deletion site is shown in lower-case letters, while the $3^{\prime}$ region is in upper-case letters (color figure online)

account for frequently seen cross-contamination during the sequencing process (Tosar et al. 2014; Zhang et al. 2012), were found in the remaining 17 exons at the $3^{\prime}$ end of the gene (Fig. 1b). A number of sequencing reads aligned to a 525-bp long region upstream of Glyma05g08150, which was adjacent to the other border of the genome region. In contrast to N0304-303-3, a significantly higher number of RNA reads from all other genotypes aligned to most exons in the region, but not to the intergenic sequence between Glyma05g08140 and Glyma05g08150 (Fig. 1b). Those data suggest that a large genomic segment was deleted 
between exon 8 of Glyma05g07926 and a short region upstream of Glyma05g08150 in N0304-303-3 (Fig. 1c).

To verify the putative deletion on chromosome 5 , we carried out a genomic PCR analysis using primers that flank the predicted deletion site in N0304-303-3 (see Fig. 1c for approximate primer location). As expected, DNA from N0304-303-3 produced an 805-bp PCR product, while DNA from the control genotype failed to produce any PCR products because the DNA fragment between both primers was too long to be amplified. Sequencing of the PCR product revealed that a 254,049-bp long genomic region was deleted between Gm5:7,846,734 and Gm5:8,100,782. No change has been detected in the sequences flanking the deletion site (Fig. 1d).

The sequencing reads found in the intergenic region downstream of the $3^{\prime}$ deletion site suggest that Glyma05g07926 was transcribed across the deletion junction thereby creating a novel transcript consisting of the 5' sequence of Glyma05g07926, the intergenic region upstream of Glyma05g08150, and Glyma05g08150 (Fig. 1c). We also identified two RNA read pairs in N0304303-3 that spanned the large DNA deletion (data not shown), which provided additional evidence for the creation of a fusion gene. The Glyma05g07926 gene encodes a TBP-ASSOCIATED FACTOR 2, which is involved in the RNA transcription machinery. It will be interesting to determine whether the new fusion transcript has a biological function.

\section{The 254-kb deletion containing the FATB1a gene and was correlated with low palmitic acid levels in N0304-303-3 and its progenitors}

We showed that the deleted region in N0304-303-3 contained a FATB1 $a$ gene (Glyma05g08060) encoding a fatty acyl-ACP thioesterase B (Fig. 2; Table 2). The enzyme hydrolyzes palmitic acid-ACP to produce palmitic acid in the de novo fatty acid biosynthetic pathway (Suppl. Figure 2) (Li-Beisson et al. 2013). Consequently, the deletion of the FATBla gene might cause the reduced accumulation of palmitic acid observed in N0304-303-3. Two sets of PCR primers were designed to detect the $254-\mathrm{kb}$ deletion and the FATB1a gene in 11 extant soybean genotypes involved in the development of N0304-303-3. PCR amplification should produce an 805-bp long DNA fragment from genotypes carrying the 254-kb deletion, and a 530-bp DNA fragment from genotypes containing the FATB1a gene. The PCR analysis revealed that N69-2774, PI 90406-1, PI 92567, PI 123440, C1726, cv. Soyola, N97$3363-4$, and cv. Brim produced the PCR amplicon expected from the FATBla gene. None of eight genotypes produced the PCR amplicon expected from the deletion (Fig. 2a), suggesting that the eight genotypes were homozygous
Fig. 2 Origin of the large deletion discovered in N0304-303-3. a Extant soybean genotypes associated with the development of N0304-303-3 were screened by PCR for presence of the large deletion and the FATBla gene. If the 254-kb deletion is present in one or both (diploid) chromosomes 5 , then an 805 bp product will be amplified. If the FATB1a gene is present, either in haploid or diploid condition, then a 530 bp product will be amplified. The presence/absence of the deletion and FATB1a in homozygous or heterozygous condition was deduced from both PCR results (see summary table). b A pedigree of genotypes involved in the development of N0304-303-3 is presented together with their deletion genotype (see a) and their palmitic acid content. Genotypes in a red box contain the deletion, while genotypes in a green box lack the deletion. A white font in a red box indicates a genotype with a homozygous deletion, whereas a black font in a red box represents genotypes with a heterozygous deletion. The palmitic acid content is given after the genotype name as normal $(N)$, intermediate $(I)$, or a low $(L)$ (color figure online)

for both presence of FATBIa and absence of the deletion. N79-2077-12 produced PCR products from both primer sets, indicating that the genotype contains the deletion and FATB1a in heterozygous condition (Fig. 2a). N0304303-3 and N97-3690 produced the expected PCR product from the deletion-flanking region, but not for the FATB $1 a$ gene, indicating that they were homozygous for the $254-\mathrm{kb}$ deletion. In addition, we measured the palmitic acid content in all genotypes, and showed that the $254-\mathrm{kb}$ deletion in homozygous and heterozygous condition was correlated with low palmitic acid levels. Genotypes homozygous for FATBla produced palmitic acid at an average level of $9.8 \%$ (Fig. 2a), which is comparable to commodity soybean oil. The hemizygous FATB1a genotype (N97-207712) accumulated $5.4 \%$ of palmitic acid while the homozygous deletion genotypes, N97-3690 and N0304-303-3, had an average of $4.2 \%$. It is possible that the zygosity status of the deletion affects the palmitic acid content. We detected the 254-kb deletion in N79-2077-12, but not in any of its three progenitors (PI 90406-1, PI 92567 and N69-2774) (Fig. 2b). This suggests that the 254-kb deletion may have occurred during the development of N79-2077-12 from those progenitor genotypes. The deletion was then most likely transmitted from genotype N79-2077-12 through N97-3690 to N0304-303-3. The PCR primers designed to amplify FATB1a and the sequences flanking the 254-kb deletion can be used in molecular breeding to select for a low palmitic acid phenotype in segregating populations.

We also observed that the first intron of the KASIIIA gene (Glyma09g41380) was not spliced out in N0304303-3 (Fig. 3). This resulted in a premature stop codon that likely renders the encoded 3-ketoacyl-ACP synthase III nonfunctional. Transcript accumulation of KASIIIA was 1.7-fold higher in N0304-303-3 than the average of the other genotypes. The intron retained in the KASIIIA transcript had a nucleotide change from GT to AT in its $5^{\prime}$ splicing consensus sequence. The same nucleotide change was reported previously in the $f a p_{1}$ allele of $\mathrm{C} 1726$ (Cardinal 
a

\begin{tabular}{|c|c|c|c|c|c|}
\hline Gel lane & Genotype & $\begin{array}{c}\mathbf{2 5 4 - k b} \text { deletion } \\
\text { product }\end{array}$ & $\begin{array}{c}\text { FATB1a } \\
\text { product }\end{array}$ & $\begin{array}{c}\mathbf{\%} \text { Seed } \\
\mathbf{1 6 : 0}\end{array}$ & Interpretation for genotypes \\
\hline 1 & N69-2774 & No & Yes & 11.0 & No deletion, Homozygous FATB1a \\
\hline 2 & PI 90406-1 & No & Yes & 11.2 & No deletion, Homozygous FATB1a \\
\hline 3 & PI 92567 & No & Yes & 10.2 & No deletion, Homozygous FATB1a \\
\hline 4 & N79-2077-12 & Yes & Yes & 5.4 & Heterozygous deletion, Heterozygous FATB1a \\
\hline 5 & Pl 123440 & No & Yes & 9.6 & No deletion, Homozygous FATB1a \\
\hline 6 & C1726 & No & Yes & 8.5 & No deletion, Homozygous FATB1a \\
\hline 7 & cv. Soyola & No & Yes & 10.7 & No deletion, Homozygous FATB1a \\
\hline 8 & N97-3690 & Yes & No & 4.4 & Homozygous deletion, No FATB1a \\
\hline 9 & N97-3363-4 & No & Yes & 7.7 & No deletion, Homozygous FATB1a \\
\hline 10 & N0304-303-3 & Yes & No & 4.0 & Homozygous deletion, No FATB1a \\
\hline 11 & cv. Dare & No & Yes & 10.1 & No deletion, Homozygous FATB1a \\
\hline 12 & cv. Brim & No & Yes & 12.0 & No deletion, Homozygous FATB1a \\
\hline
\end{tabular}

254-kb Deletion

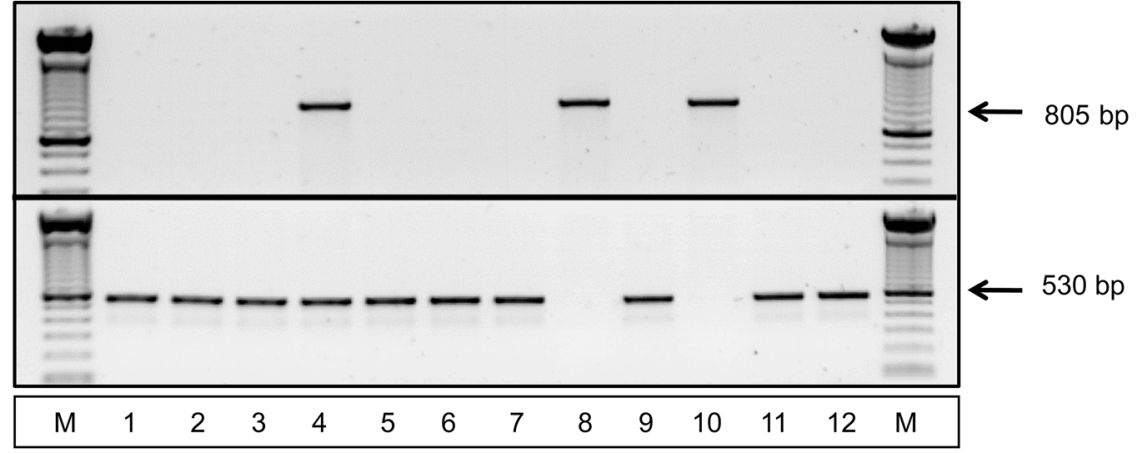

FATB1a

b

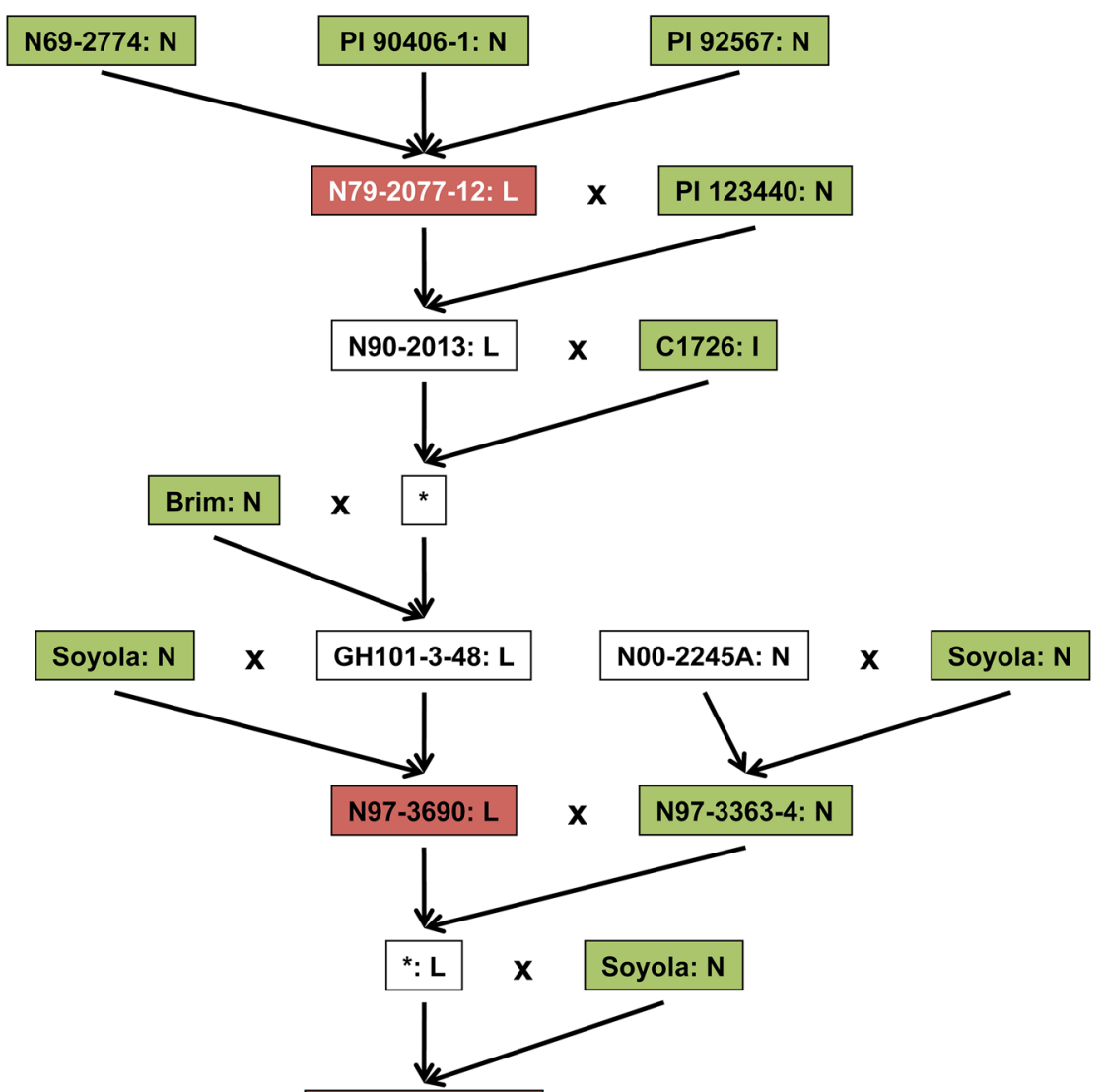

N0304-303-3: L 


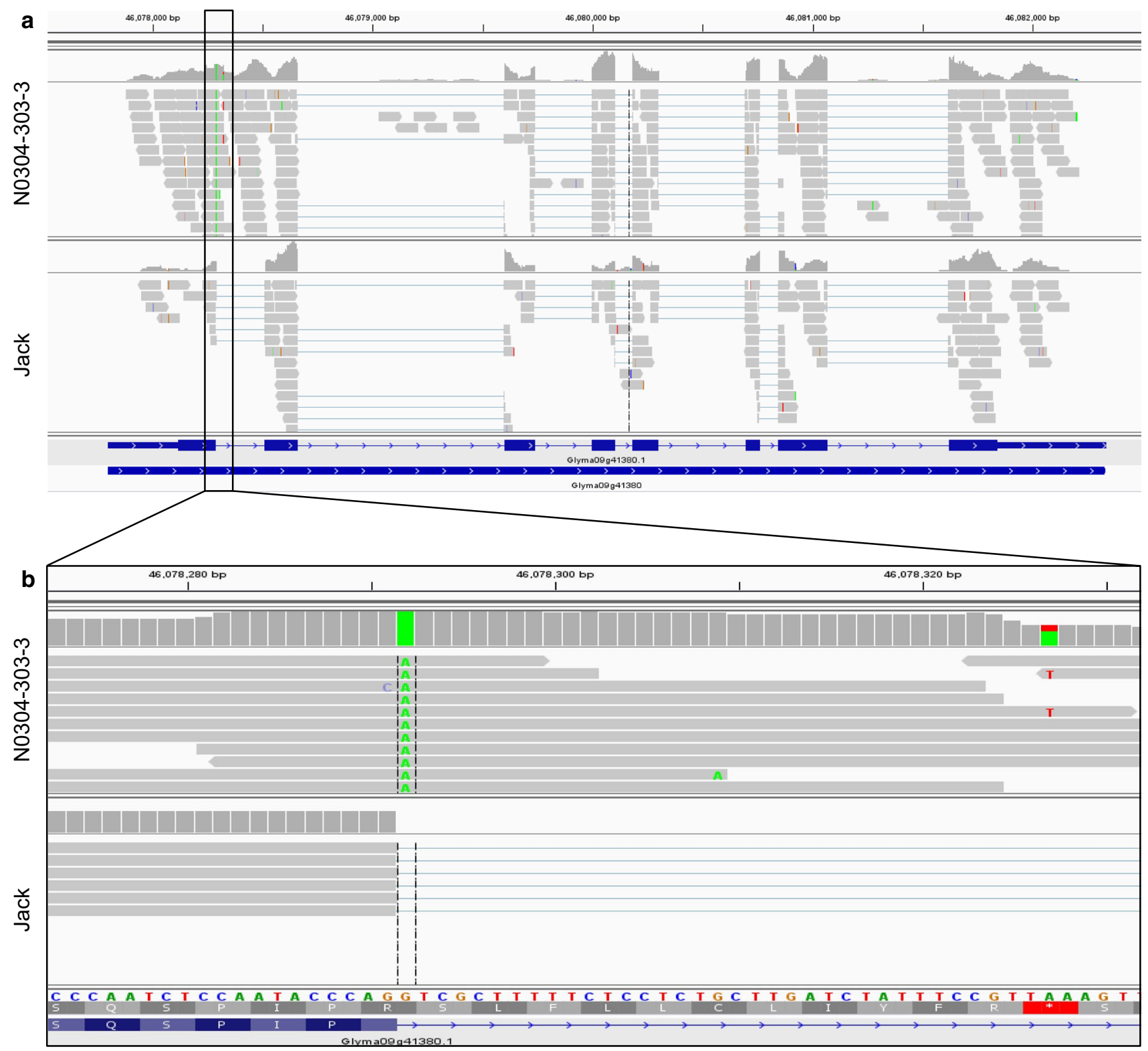

Fig. 3 IGV view of the KASIIIA gene encoding a 3-ketoacyl ACP synthase. a RNA-seq reads for N0304-303-3 and Jack aligning to the KASIIIA gene model are shown. Please note that RNA-seq reads align to the first intron of N0304-303-3 while no reads can be detected in the intron for Jack. b A zoomed-in view of the first exon-intron junc-

et al. 2014). C1726 was also involved in the development of N0304-303-3. It is likely that the KASIIIA allele was introduced into N0304-303-3 from C1726. C1726, which does not contain the large genomic deletion containing the FATBIa gene, had an intermediate palmitic acid level of $8.5 \%$ (Fig. 2a). The mutated KASIIIA allele potentially contributes to additional reduction of palmitic acid levels in N0304-303-3 (4.0\% of palmitic acid) compared to N792077-12 (5.2\% of palmitic acid). However, its effects on tion reveals a single nucleotide change from $\mathrm{G}$ to $\mathrm{A}$ at the $5^{\prime}$ splice consensus sequence in N0304-303-3. The SNP may have prevented the splicing of intron 1 in N0304-303-3. Retention of the first intron results in a reading frame shift subsequently leading to a premature stop codon (shown in red) (color figure online)

the accumulation of palmitic acid in the presence of the 254-kb deletion remain to be determined.

\section{The 254-kb deletion was located in a functionally conserved duplicated chromosome segment}

The 254-kb deleted region was located on a duplicated genome segment retained from the Glycine-specific whole genome duplication that occurred 13 million years ago. 


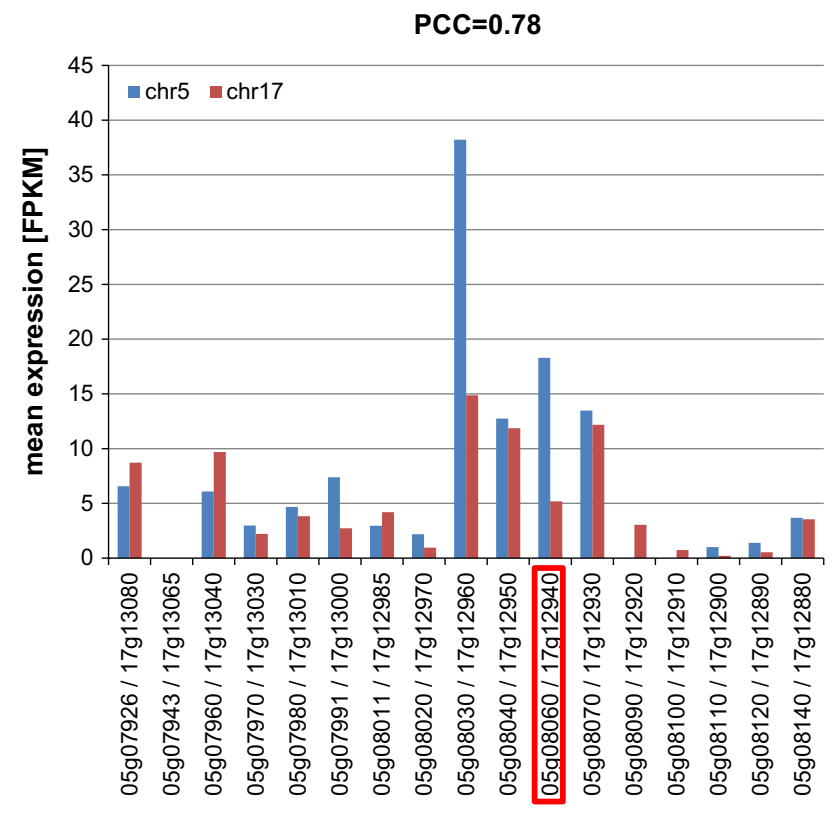

Fig. 4 Expression of genes in the N0304-303-3 deleted region. The average expression of genes in the N0304-303-3 deleted region and their homoeologs, which are derived from eight soybean genotypes, are displayed. Gene annotations and additional information are listed in Table 2. The gene pair framed in red refers to FATB1a and FATB1b (color figure online)

Table 3 Expression of FATB genes (in FPKM)

\begin{tabular}{llcrrr}
\hline Gene & Gene ID & N0304-303-3 & Mean & Stdev & $(\%)$ \\
\hline FATB1a & Glyma05g08060 & 0.11 & 18.30 & 4.1 & 25.80 \\
FATB1b & Glyma17g12940 & 5.52 & 5.14 & 1.3 & 7.24 \\
FATB2 $a$ & Glyma04g21910 & 26.79 & 44.13 & 40.3 & 62.22 \\
FATB2 $b$ & Glyma06g23560 & 2.88 & 3.37 & 2.8 & 4.75 \\
\hline
\end{tabular}

The genes in the duplicated regions were highly syntenic (Suppl. Figure 2). Each of the 18 annotated gene models in the deleted region had a corresponding homoeologous gene in its duplicated region on chromosome 17. Seventeen of the eighteen annotated gene models in the deletion region are likely functional genes. Gene models Glyma05g08080/ Glyma17g12925 shared similarities in their DNA sequence, but no protein identity. Neither of those two gene models was expressed in seeds. They were likely pseudo genes and were omitted from further analysis (Table 2).

We calculated the synonymous substitution rates $K_{\mathrm{s}}$, the non-synonymous substitution rates $K_{\mathrm{a}}$ and the $K_{\mathrm{a}} / K_{\mathrm{s}}$ ratio for the 17 duplicated gene pairs, which revealed high similarities in their coding sequence alignments. The $K_{\mathrm{s}}$ values of these 17 genes in the deleted regions ranged from 0.06 to 0.22 with an average of 0.13 (Table 2). These values are consistent with genes that emerged from the most recent genome duplication event 13 million years ago (Lin et al.
2010; Roulin et al. 2012; Schmutz et al. 2010). The $K_{\mathrm{a}} / K_{\mathrm{s}}$ ratios for the homoeologous genes ranged from 0.09 to 0.67 with an average of 0.30 , which was much less than 1 , suggesting that the homoeologous genes underwent a strong purifying selection. The proteins encoded by those duplicated genes had a high sequence similarity with an average of $91.6 \%$ (Table 2), indicating that they may have identical or similar protein activities.

We examined the expression of the 17 homoeologous gene pairs in soybean seeds based on our transcriptome sequencing data. Fourteen of these 17 genes in the deleted region were expressed in seeds of the other examined genotypes, which did not contain the genomic deletion (Table 2). Interestingly, all of their corresponding homoeologous genes on chromosome 17 were expressed in seeds as well. Those gene pairs were transcribed at similar levels with an overall Pearson's correlation coefficient (PCC) value of 0.78 (Fig. 4). Three of the seventeen genes were not expressed in seeds. However, two of their homoeologous genes on chromosome 17 were expressed in seeds, suggesting that their expression patterns diverged after the whole genome duplication.

Soybean contains four genes encoding fatty acyl-ACP thioesterases B. FATBla was highly expressed in soybean seeds at a level of 18.3 FPKM. Its homoeologous gene on chromosome 17, FATB1b (Glyma17g12940), was expressed at a lower level of 5.18 FPKM. FATB1a and FATB $1 b$ shared $98.3 \%$ protein sequence similarity, strongly suggesting that both FATB1a and FATB1b have similar enzymatic functions. In addition, FATB $1 a$ and $F A T B 1 b$ had a very low $K_{\mathrm{a}} / K_{\mathrm{s}}$ value of 0.18 , indicating that they were subject to a strong purifying selection. The other FATB genes, FATB2a (Glyma04g21910) and $F A T B 2 b$ (Glyma06g23560) were also abundantly expressed in soybean seeds at levels of 44.1 and 3.4 FPKM, respectively (Table 3). FATB1a shared 76.4 and $76.2 \%$ protein similarity with FATB2a and FATB2b respectively, suggesting that they potentially share similar enzymatic functions with FATB1a. Thus, the deletion of FATBla probably did not result in a complete loss of fatty acyl-ACP thioesterases B activities in soybean seeds. The remaining $4 \%$ palmitic acid content observed in N0304-303-3 is likely attributed to the activities of the three functional FATB genes.

\section{The large deletion in M23 was also located in a functionally conserved duplicated region}

As predicted above, a large down-regulated gene cluster mapped to a previously reported 164-kb deletion in M23 (Bolon et al. 2011). While this deletion was previously reported, the expression of genes in the deleted region and their evolutionary relationship with their homoeologous genes has not been characterized. Like the large deletion in 


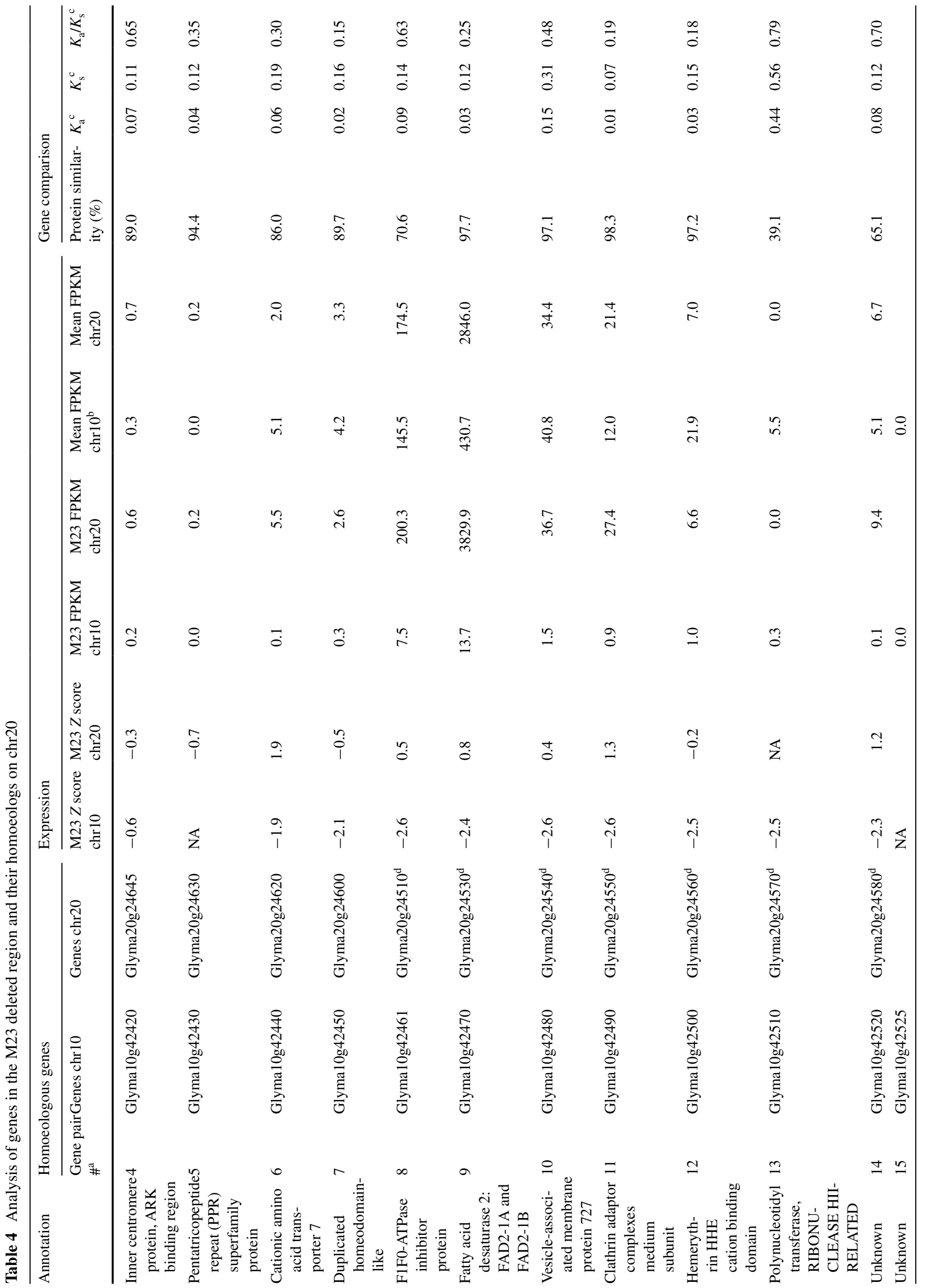




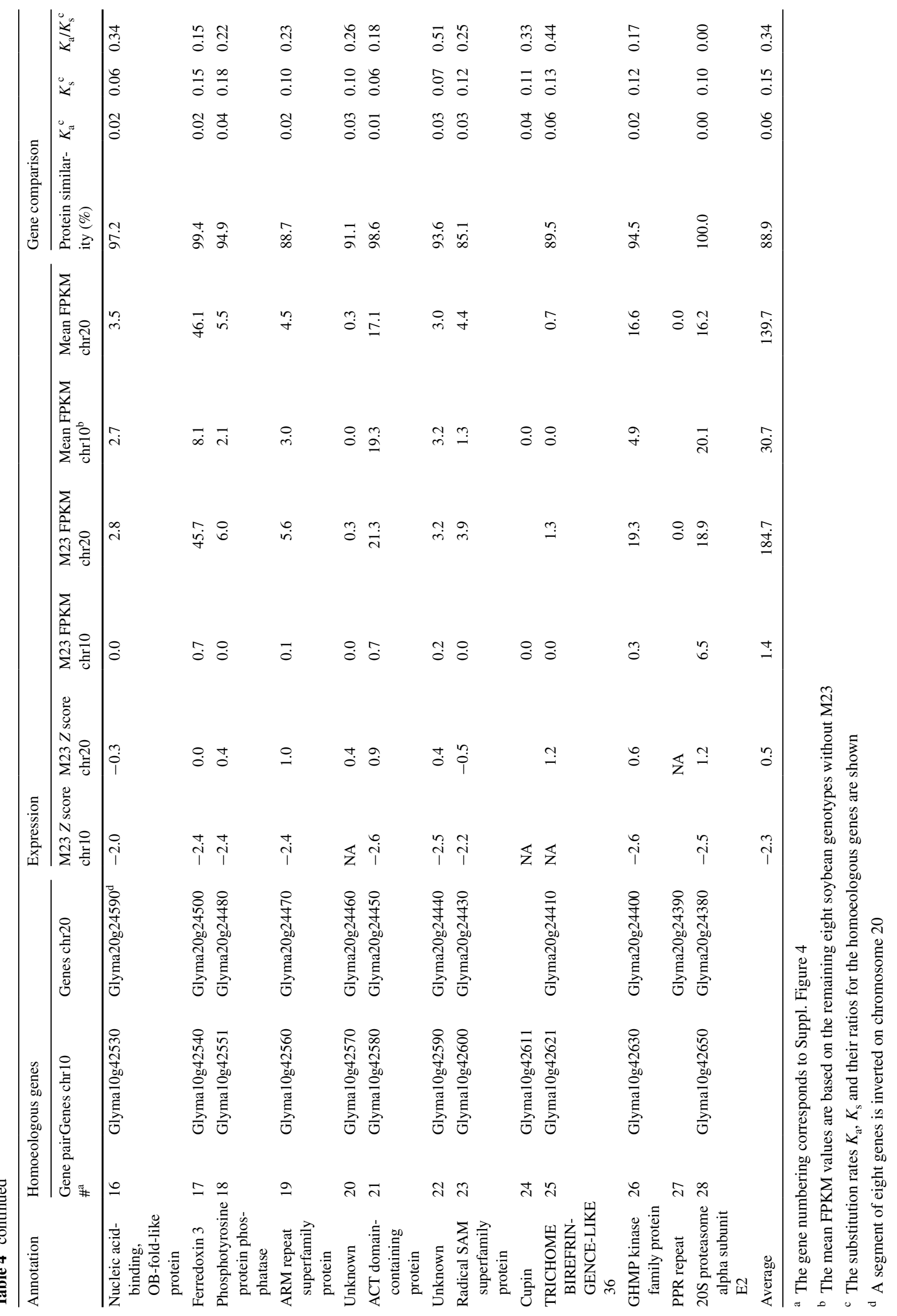


N0304-303-3, we observed that the deleted region in M23 was also located on a duplicated chromosome segment retained from the Glycine-specific whole genome duplication (Table 4; Suppl. Figure 4). Out of the 24 genes in the deleted region, 22 had homoeologs in a duplicated region on chromosome 20 (Table 4). The homoeologous gene pairs had an average $K_{\mathrm{a}} / K_{\mathrm{s}}$ ratio of 0.34 and their encoded proteins had an average sequence similarity of $88.95 \%$ (Table 4), suggesting that the genes in the M23 deletion also underwent purifying selection and are most likely subject to functional constrains. Nineteen out of twenty-four genes in the M23-deleted region were expressed in seeds (Table 4). The majority of the 18 homoeologous genes were expressed in seeds at similar levels with a PCC value of 0.96 (Table 4; Suppl. Figure 5).

The deleted region contains the FAD2-1A gene (Glyma10g42470), which encodes one of two fatty acid desaturases 2 responsible for converting oleic acid into linoleic acid in seeds. It was shown that the loss of FAD2-1A results in the mid-oleic acid phenotype of M23 (Alt et al. 2005; Sandhu et al. 2007). FAD2-1A and its homoeologous gene $F A D 2-1 B$ on chromosome 20 were highly expressed in seeds. Their proteins shared $97.7 \%$ sequence similarity (Table 4). The deletion of FAD2-1A in M23 only reduces, it does not completely abolish the ability of M23 to catalyze the desaturation of oleic to linoleic acid during fatty acid biosynthesis.

\section{Discussion}

Genome sequencing is widely used to characterize genome structure variations. Recently, we successfully applied transcriptome sequencing technologies to identify expression variation and small-scale genome sequence changes such as SNPs and 1 or 2-bp indels in nine soybean genotypes. In this study, we identified a total of nine genomic regions containing genotype-specific co-regulated gene clusters in soybean seeds. Out of the four down-regulated genomic regions, one was mapped to a previously identified large deletion in M23. We demonstrated that two down-regulated regions in N0304-303-3 were caused by a novel 254$\mathrm{kb}$ deletion. One of the up-regulated genomic regions in Jack mapped to the Rhgl locus, which is consistent with our previous observation that the copy number of the four genes in the Rhgl locus are amplified by about tenfold in Jack (Goettel et al. 2014). Thus, all of the four examined co-regulated gene clusters/genome regions were associated with large genome structural variation, demonstrating the high effectiveness of the approach to predict large-scale genome rearrangements. It will be interesting to investigate if the remaining co-regulated gene clusters also concur with large structural variation. In addition, the transcriptome sequencing approach revealed a transcribed fusion gene in N0304-303-3 that was created by the large deletion event. Collections of soybean mutants such as fast-neutron induced mutants containing large genome structural variation have been generated by the soybean community. Comparative genomic hybridization and genome sequencing have been successfully used to identify and characterize the large genome structural variation in those soybean mutants (Bolon et al. 2011). While transcriptome sequencing has been widely used to study global gene transcriptional regulation, its application for the discovery of largescale genome structural variation in those soybean mutants could be further explored. In summary, the transcriptome sequencing approach allows us to effectively predict largescale genome structural variation, and also provides insight into resulting transcriptional changes at the molecular and systems levels.

We showed that the 254-kb deletion was associated with a reduction of palmitic acid in N0304-303-3 and the genotypes involved in the development of N0304-303-3, suggesting that the low palmitic acid content was caused by the large deletion. The deleted region contains the FATB la gene, which encodes a fatty acyl-ACP thioesterase B. This enzyme terminates the fatty acyl chain extension during de novo fatty acyl synthesis by hydrolyzing acyl groups and releasing free palmitic acid (Li-Beisson et al. 2013). Also, non-functional FATBla alleles have been shown to result in lower palmitic acid levels (Cardinal et al. 2007, 2014). Therefore the loss of the FATB1a enzymatic activity is likely responsible for the decrease of palmitic acid levels in N0304-303-4. Interestingly, N79-2077-12, which carries the $f_{a p} p_{n c}$ allele, was involved in developing N0304-303-3. Our analysis showed that N79-2077-12 contained the 254-kb deletion, suggesting that the 254-kb deletion represents the molecular basis underlying the $f a p_{n c}$ allele. The large-scale deletion also explains a previous report that Southern, northern and cDNA analysis failed to detect FATBIa in $f_{a p}$ genotypes (Cardinal et al. 2007; Wilson et al. 2001a, c). The three genotypes used to develop N79-2077 lacked the 254-kb deletion and had normal palmitic acid levels. One of those genotypes, N69-2774, is an ms $1 m s 1$ male-sterile maintainer genotype (Brim and Young 1971). Genetic crosses with it are known to induce instability during meiosis. Thus, the initial mating with N69-2774 likely caused the 254-kb deletion in developing N79-2077. The identification of the deletion junction site and the new fusion transcript created by the deletion allows us to develop molecular markers to detect the $254-\mathrm{kb}$ deletion allele at both DNA and RNA levels for a variety of applications.

We also detected another low palmitic acid mutant allele, $\mathrm{fap}_{1}$, in N0304-303-3 in addition to the FATBIa 
deletion (Cardinal et al. 2014). We observed the missplicing of the first intron of the KASIIIA pre-mRNA and a single nucleotide mutation at the exon1-intron1 splice junction of the KASIIIA transcript. This is consistent with the results from a previous characterization of $f a p_{1}$ in the EMS mutant genotype C1726 (Cardinal et al. 2014). C1726 was used in the development of N0304-303-3, and is probably the source of the $f a p_{1}$ allele in N0304-303-3 (Fig. 2b). We did not detect the 254-kb deletion in C1726 (Fig. 2a). C1726 contains an intermediate level of palmitic acid. Genetic analysis showed that there were significant epistatic interactions between $f a p_{1}$ and $f a p_{n c}$ (Cardinal et al. 2014). Their epistatic interaction may be caused by the fact that KASIIIA catalyzes the initial condensation reaction of acetyl-CoA and malonyl-ACP upstream of FATB1a in the fatty acid biosynthetic pathway.

Besides lower palmitic acid levels, N0304-303-3 also has a significantly reduced stearic acid and elevated oleic acid content. It may partially be caused by the KASIIIA mutation. However, it has previously been reported that genotypes homozygous for $f_{a p} p_{n c}$ have decreased stearic acid levels (Cardinal et al. 2007; Rebetzke et al. 1998, 2001). In Arabidopsis, FATB thioesterases showed significant activity with 18:1-ACP and 18:0-ACP (Salas and Ohlrogge 2002), which is consistent with the reduction of stearic acid seen in N0304-303-3. The increased oleic acid content in N0304-303-3 may be caused by the redirection from synthesizing palmitic and stearic acid into oleic acid synthesis (Upchurch and Ramirez 2010).

We showed that all four FATB genes encoded proteins with high sequence similarity and were expressed at significant levels in seeds, suggesting that palmitic acid production is controlled by all four FATB genes. As expected, the deletion of FATBla did not entirely eliminate the production of palmitic acid in N0304-303-3, it did, however, reduce it by about $60 \%$. The remaining palmitic acid suggests that one or more of the three additional FATB thioesterases contribute to the palmitic acid production. This reduction in palmitic acid, however, was not fully correlated with the FPKM expression values from the FATB genes at mid-maturation stage (Table 3). Mutants for $F A T B 1 b, F A T B 2 a$ and $F A T B 2 b$ are required to determine their individual contribution to the total palmitic acid content. The knowledge about the expression and structural variation of the $F A T B$ genes and alleles should be valuable for breeders to design crossing and selection strategies to modify palmitic acid production for new cultivar development.

The large-scale deletions in both M23 and N0304-303-3 were located in duplicated genome segments retained from the Glycine-specific whole genome duplication. Expression patterns and protein coding sequences of duplicated genes evolve rapidly if they are not subject to functional constraints (An et al. 1996a, b; Gu et al. 2002). All homoeologous gene pairs in both large-scale deletions had $K_{\mathrm{a}} / K_{\mathrm{s}}$ values smaller than 1 , indicating that those genes are subject to a strong purifying selection and have conserved protein functions. Duplicated genes that acquire new functions (neo-functionalization) are the outcome of positive selection pressure, that is, their $K_{\mathrm{a}} / K_{\mathrm{s}}>1$. Interestingly, all homoeologous gene pairs in both deletions have $K_{\mathrm{a}} / K_{\mathrm{s}}$ values smaller than 1 , indicating that these genes did not gain new functions after the Glycine-specific duplication event. Most of the duplicated genes in the deleted region had similar expression levels. However, since the expression of those homoeologous genes in the deleted region was only measured in seeds at the mid-maturation stage, subfunctionalization based on divergent expression patterns in other tissues and developmental stages is plausible for the homoeologous genes after the duplication event. Conservation of gene function and/or sub-functionalization in gene expression due to a strong purifying selection pressure are supported by $K_{\mathrm{a}} / K_{\mathrm{s}}$ ratios $<1$.

The functional conservation of duplicated genes could explain the lack of lethal or null phenotypes (i.e., the phenotype in the absence of redundant gene functions) in genotypes containing such large-scale deletions. Almost all genes in each large-scale deletion encoded functional proteins and were expressed at a significant level in seeds. We propose that the loss of so many functionally significant genes in such large deletions does not cause lethal or null phenotypes for M23 and N0304-303-3, because their functionally conserved homoeologous genes partially or fully complement the deleted genes. Any phenotype that is the result of gene products from two or more functionally conserved homoeologs may be subject to a gene dosage effect upon deletion of one or more individual homoeologs. For example, the deletion of the FATBIa gene reduces palmitic acid levels by about $60 \%$ in N0304-303-3 but does not eliminate it. Likewise, the deletion of FAD2-1A does not completely block the conversion of oleic to linoleic acid since the linoleic acid content is only decreased by about $55 \%$ (Suppl. Figure 1). Accordingly, both deletions described here may represent impressive examples of gene dosage effects in soybean. This hypothesis is consistent with a recent report of a 206-kb deletion in the high palmitic acid genotype $\mathrm{J} 10$ containing the KASIIA gene along with 16 other genes. That deleted region is also located on a duplicated chromosome segment. No notable growth defects were detected in J10 plants under field conditions (Anai et al. 2012).

Our observations for both deleted regions were consistent with the result from a genome-wide analysis of paralogs in soybean. A study of 8910 strictly duplicated gene pairs revealed that only a few duplicates were neo- or non-functionalized (Roulin et al. 2012). It has also been 
reported that the pseudogenization rate for duplicates within a $1 \mathrm{mb}$ homoeologous soybean regions was less than that of single copy genes (Lin et al. 2010). This suggests that large deletions can occur and could be viable in many other duplicated genome segments. It also raises the possibility that large non-deleterious deletions derived from mutagenesis experiments may preferentially be located in duplicated genome segments and are less likely generated in single copy regions.

While in genetic theory duplicates are evolutionarily unstable, it has been shown in Arabidopsis and rice that higher impact non-synonymous changes occurred less frequently in duplicated genes than in single copy genes (Chapman et al. 2006). Evidentially, functional buffering between duplicates can persist for tens of millions of years (Wang et al. 2012). The retention of highly conserved homoeologous genes for 13 million years after the whole Glycine-genome duplication should offer an evolutionary advantage for soybean. Accordingly, the loss of a conserved homoeologous gene, as seen for example in large deletions, should come with reduced fitness. Although none of those large deletions result in detrimental phenotypic changes, they appear to have an effect on soybean fitness. In addition to the fatty acid composition changes in M23 and N0304-303-3, it has been reported that the $f a p_{n c}$ allele, which is caused by the $254-\mathrm{kb}$ deletion, is associated with decreased yield and reduction in plant height (Cardinal et al. 2014). The M23 deletion appears to affect plant growth and development (unpublished). Thus, it seems that some of the deleted genes were not fully complemented by their homoeologs. Although natural selection may be of less concern for a crop species, selection by breeders will not favor the maintenance of a deletion in a soybean genotype with lower yield potential. The molecular and functional genomics characterization of the large deletions in N0304-303-3 and M23 offers an excellent example for genetic redundancy and dosage effects of homoeologous genes on agronomic traits, which are important in soybean genetics and breeding. Breeders may need to combine mutant alleles of homoeologous genes to develop cultivars with significantly improved target traits (Pham et al. 2010). Eventually, dosage effects may provide excellent opportunities for breeders to control a target trait quantitatively.

Author contribution statement WG and YQA conceived of the study, participated in the design and data analysis of the study. RGU provided seeds for various soybean genotypes. MR and RGU participated in the genomic PCR analysis and the seed fatty acid composition analysis. RGU provided important pedigree information for genotype N0304-303-3. YQA oversaw and coordinated the study. WG, YQA and RGU wrote the manuscript.
Acknowledgments The authors would like to thank Rick Meyer and William Novitzky for their excellent technical assistance, data analysis and valuable comments on the manuscript, and Dr. Joe Burton for his help in pedigree analysis. The research is funded by the United Soybean Board (USB Project \#: 1420-632-6607) and USDA-ARS (Project \#: 3622-21000-037-00) to YQA.

\section{Compliance with ethical standards}

Disclaimer note Names are necessary to report factually on available data; however, the USDA neither guarantees nor warrants the standard of the product, and the use of the name by USDA implies no approval of the product to the exclusion of others that may also be suitable. USDA is an equal opportunity provider and employer.

Conflict of interest The research is funded by the United Soybean Board (USB Project \#: 1420-632-6607) and USDA-ARS (Project \#: 3622-21000-037-00) to YQA. The authors declare that they have no conflict of interest.

Ethical standards The experiments performed for the publication comply with the current laws of the United States of America.

Open Access This article is distributed under the terms of the Creative Commons Attribution 4.0 International License (http://creativecommons.org/licenses/by/4.0/), which permits unrestricted use, distribution, and reproduction in any medium, provided you give appropriate credit to the original author(s) and the source, provide a link to the Creative Commons license, and indicate if changes were made.

\section{References}

Alt J, Fehr W, Welke G, Sandhu D (2005) Phenotypic and molecular analysis of oleate content in the mutant soybean line M23. Crop Sci 45:1997-2000

An YQ, Huang S, McDowell JM, McKinney EC, Meagher RB (1996a) Conserved expression of the Arabidopsis ACT1 and ACT 3 actin subclass in organ primordia and mature pollen. Plant Cell 8:15-30

An YQ, McDowell JM, Huang S, McKinney EC, Chambliss S, Meagher RB (1996b) Strong, constitutive expression of the Arabidopsis ACT2/ACT8 actin subclass in vegetative tissues. Plant $\mathbf{J}$ 10:107-121

Anai T, Hoshino T, Imai N, Takagi Y (2012) Molecular characterization of two high-palmitic-acid mutant loci induced by X-ray irradiation in soybean. Breed Sci 61:631-638

Bolon Y-T, Haun WJ, Xu WW, Grant D, Stacey MG, Nelson RT, Gerhardt DJ, Jeddeloh JA, Stacey G, Muehlbauer GJ, Orf JH, Naeve SL, Stupar RM, Vance CP (2011) Phenotypic and genomic analyses of a fast neutron mutant population resource in soybean. Plant Physiol 156:240-253

Brim CA, Young MF (1971) Inheritance of a male-sterile character in soybeans. Crop Sci 11:564-566

Burkey KO, Booker FL, Pursley WA, Heagle AS (2007) Elevated carbon dioxide and ozone effects on peanut: II. Seed yield and quality. Crop Sci 47:1488-1497

Burton J, Wilson R, Brim C (1994) Registration of N79-2077-12 and N87-2122-4, two soybean germplasm lines with reduced palmitic acid in seed oil. Crop Sci 34:313

Cardinal A, Burton J, Camacho-Roger A, Yang J, Wilson R, Dewey R (2007) Molecular analysis of soybean lines with low palmitic acid content in the seed oil. Crop Sci 47:304-310 
Cardinal AJ, Whetten R, Wang S, Auclair J, Hyten D, Cregan P, Bachlava E, Gillman J, Ramirez M, Dewey R, Upchurch G, Miranda L, Burton JW (2014) Mapping the low palmitate fap1 mutation and validation of its effects in soybean oil and agronomic traits in three soybean populations. Theor Appl Genet 127:97-111

Carver TJ, Rutherford KM, Berriman M, Rajandream MA, Barrell BG, Parkhill J (2005) ACT: the Artemis comparison tool. Bioinformatics 21:3422-3423

Chapman BA, Bowers JE, Feltus FA, Paterson AH (2006) Buffering of crucial functions by paleologous duplicated genes may contribute cyclicality to angiosperm genome duplication. Proc Natl Acad Sci USA 103:2730-2735

Clemente TE, Cahoon EB (2009) Soybean oil: genetic approaches for modification of functionality and total content. Plant Physiol 151:1030-1040

De Vries BD, Fehr WR, Welke GA, Dewey RE (2011) Molecular characterization of the mutant fap3 (A22) allele for reduced palmitate concentration in soybean. Crop Sci 51:1611-1616

Durrett TP, Benning C, Ohlrogge J (2008) Plant triacylglycerols as feedstocks for the production of biofuels. Plant J 54:593-607

Erickson EA, Wilcox JR, Cavins JF (1988) Inheritance of altered palmitic acid percentage in two soybean mutants. J Hered 79:465-468

Fehr WR, Welke GA, Cianzio SR, Duvick DN, Hammond EG (1991) Inheritance of reduced palmitic acid content in seed oil of soybean. Crop Sci 31:88-89

Goettel W, Xia E, Upchurch R, Wang ML, Chen P, An YQ (2014) Identification and characterization of transcript polymorphisms in soybean lines varying in oil composition and content. BMC Genom 15:299

Gu Z, Nicolae D, Lu HH-S, Li W-H (2002) Rapid divergence in expression between duplicate genes inferred from microarray data. Trends Genet 18:609-613

Li-Beisson Y, Shorrosh B, Beisson F, Andersson MX, Arondel V, Bates PD, Baud S, Bird D, Debono A, Durrett TP, Franke RB, Graham IA, Katayama K, Kelly AA, Larson T, Markham JE, Miquel M, Molina I, Nishida I, Rowland O, Samuels L, Schmid KM, Wada H, Welti R, Xu C, Zallot R, Ohlrogge J (2013) Acyllipid metabolism. Arabidopsis Book 11:e0161

Lin JY, Stupar RM, Hans C, Hyten DL, Jackson SA (2010) Structural and functional divergence of a $1-\mathrm{Mb}$ duplicated region in the soybean (Glycine max) genome and comparison to an orthologous region from Phaseolus vulgaris. Plant Cell 22:2545-2561

Nei M, Gojobori T (1986) Simple methods for estimating the numbers of synonymous and nonsynonymous nucleotide substitutions. Mol Biol Evol 3:418-426

Ozsolak F, Milos PM (2011) RNA sequencing: advances, challenges and opportunities. Nat Rev Genet 12:87-98

Pham A-T, Lee J-D, Shannon JG, Bilyeu KD (2010) Mutant alleles of FAD2-1A and FAD2-1B combine to produce soybeans with the high oleic acid seed oil trait. BMC Plant Biol 10:195

Pham A-T, Lee J-D, Shannon JG, Bilyeu KD (2011) A novel FAD2-1 A allele in a soybean plant introduction offers an alternate means to produce soybean seed oil with $85 \%$ oleic acid content. Theor Appl Genet 123:793-802

Rahman S, Takagi Y, Kubota K, Miyamoto K, Kawakita T (1994) High oleic acid mutant in soybean induced by X-ray irradiation. Biosci Biotech Biochem 58:1070-1072

Rahman S, Takagi Y, Kinoshita T (1996) Genetic analysis of palmitic acid contents using two soybean mutants, J3 and J10. Breed Sci 46:343-348

Rebetzke GJ, Burton JW, Carter TE, Wilson RF (1998) Genetic variation for modifiers controlling reduced saturated fatty acid content in soybean. Crop Sci 38:303-308
Rebetzke GJ, Pantalone VR, Burton JW, Carter TE, Wilson RF (2001) Genetic background and environment influence palmitate content of soybean seed oil. Crop Sci 41:1731-1736

Roulin A, Auer PL, Libault M, Schlueter J, Farmer A, May G, Stacey G, Doerge RW, Jackson SA (2012) The fate of duplicated genes in a polyploid plant genome. Plant J 73:143-153

Salas JJ, Ohlrogge JB (2002) Characterization of substrate specificity of plant FatA and FatB acyl-ACP thioesterases. Arch Biochem Biophys 403:25-34

Sandhu D, Alt J, Scherder C, Fehr W, Bhattacharyya M (2007) Enhanced oleic acid content in the soybean mutant M23 is associated with the deletion in the Fad2-1a gene encoding a fatty acid desaturase. J Am Oil Chem Soc 84:229-235

Schmutz J, Cannon SB, Schlueter J, Ma J, Mitros T, Nelson W, Hyten DL, Song Q, Thelen JJ, Cheng J, Xu D, Hellsten U, May GD, Yu Y, Sakurai T, Umezawa T, Bhattacharyya MK, Sandhu D, Valliyodan B, Lindquist E, Peto M, Grant D, Shu S, Goodstein D, Barry K, Futrell-Griggs M, Abernathy B, Du J, Tian Z, Zhu L, Gill N, Joshi T, Libault M, Sethuraman A, Zhang X-C, Shinozaki K, Nguyen HT, Wing RA, Cregan P, Specht J, Grimwood J, Rokhsar D, Stacey G, Shoemaker RC, Jackson SA (2010) Genome sequence of the palaeopolyploid soybean. Nature 463:178-183

Schnebly S, Fehr W, Welke G, Hammond E, Duvick D (1994) Inheritance of reduced and elevated palmitate in mutant lines of soybean. Crop Sci 34:829-833

Stijšin D, Ablett GR, Luzzi BM, Tanner JW (1998) Use of gene substitution values to quantify partial dominance in low palmitic acid soybean. Crop Sci 38:1437-1441

Takagi Y, Rahman MS, Joo H, Kawakita T (1995) Reduced and elevated palmitic acid mutants in soybean developed by X-ray irradiation. Biosci Biotech Biochem 59:1778-1779

Tamura K, Peterson D, Peterson N, Stecher G, Nei M, Kumar S (2011) MEGA5: molecular evolutionary genetics analysis using maximum likelihood, evolutionary distance, and maximum parsimony methods. Mol Biol Evol 28:2731-2739

Tosar JP, Rovira C, Naya H, Cayota A (2014) Mining of public sequencing databases supports a non-dietary origin for putative foreign miRNAs: underestimated effects of contamination in NGS. RNA 20:754-757

Upchurch R, Ramirez M (2010) Gene expression profiles of soybeans with mid-oleic acid seed phenotype. J Am Oil Chem Soc 87:857-864

Voelker T, Kinney AJ (2001) Variations in the biosynthesis of seed-storage lipids. Annu Rev Plant Physiol Plant Mol Biol 52:335-361

Wang Y, Wang X, Paterson AH (2012) Genome and gene duplications and gene expression divergence: a view from plants. Ann N Y Acad Sci 1256:1-14

Wilson RF (2012) The role of genomics and biotechnology in achieving global food security for high-oleic vegetable oil. J Oleo Sci 61:357-367

Wilson R, Burton J, Novitzky W, Dewey R (2001a) Current and future innovations in soybean (Glycine $\max$ L. Merr.) oil composition. J Oleo Sci 50:353-358

Wilson R, Marquardt T, Novitzky W, Burton J, Wilcox J, Dewey R (2001b) Effect of alleles governing 16: 0 concentration on glycerolipid composition in developing soybeans. J Am Oil Chem Soc 78:329-334

Wilson R, Marquardt T, Novitzky W, Burton J, Wilcox J, Kinney A, Dewey R (2001c) Metabolic mechanisms associated with alleles governing the 16:0 concentration of soybean oil. J Am Oil Chem Soc 78:335-340

Zhang Y, Wiggins BE, Lawrence C, Petrick J, Ivashuta S, Heck G (2012) Analysis of plant-derived miRNAs in animal small RNA datasets. BMC Genom 13:381 